\title{
Diseño DE UN CURSO DE FORMACIÓN CONTINUADA EN CUIDADOS PALIATIVOS BASADO EN COMPETENCIAS
}

\section{DESIGN OF A CONTINUOUS TRAINING PALLIATIVE CARE COURSE BASED ON COMPETENCIES}

María Oses Zubiri (1) , Alain Legault (2), Anne Marie Martinez (2)

(1) Complejo Hospitalario de Navarra. Estudiante de doctorado UPNA.

España

(2) Universidad de Montreal

Oses Zubiri, M. Legault, A. Martinez, AM (2020). DISEÑO DE UN CURSO DE FORMACIÓN CONTINUADA EN CUIDADOS PALIATIVOS BASADO EN COMPETENCIAS. Revista Ene De Enfermería, 14(1). Consultado de http://www.ene-enfermeria.org/ojs/index.php/ ENE/article/view/1021 


\section{Resumen}

El objetivo de esta publicación es mostrar la estructura y contenidos de un curso de formación continuada en cuidados paliativos para enfermería. Se han empleado las últimas tendencias metodológicas en educación, y se ha tomado como referencia el modelo de competencias de la Facultad de Enfermería de la Universidad de Montreal.

El resultado de la investigación ha conducido al diseño de un curso, del que los profesionales de la enfermería se pueden beneficiar para mejorar sus habilidades para el cuidado paliativo.

Contemplar nuevos enfoques en la formación intermedia de cuidados paliativos en enfermería, frente a los viejos métodos de aprendizaje, proporciona una mayor integración de los modelos actuales, y promueve la calidad y la satisfacción asistencial en el contexto en el que se desarrollan.

Palabras clave: Investigación en Educación de Enfermería, Educación Continua en Enfermería, Enfermería de Cuidados Paliativos al Final de la Vida.
Abstract:

The objective of this publication is to show the structure and contents of a continuing education course in palliative care for nursing. The latest methodological trends in education have been used, and the competency model of the Faculty of Nursing of the University of Montreal has been taken as reference.

The result of the research has led to the design of a course, from which nursing professionals can benefit to improve their skills for palliative care.

Contemplating new approaches in the intermediate training of palliative care in nursing, compared to the old methods of learning, provides a greater integration of current models, and promotes quality and care satisfaction in the context in which they are developed.

Key words: Nursing Education Research, Education, Nursing, Continuing, Hospice and Palliative Care Nursing 


\section{INTRODUCCIÓN}

En Europa desde 2004, y dentro del currículum de enfermería en cuidados paliativos, se distinguen tres niveles de formación por competencias, como son: el básico (pregrado), el intermedio (formación continuada) y el avanzado (especialización y posgrado) ${ }^{1}$. En España, la Asociación Española de Enfermería en Cuidados Paliativos (AECPAL) define los cursos de segundo nivel o nivel intermedio, como aquellos con una carga lectiva específica, con la que los profesionales, actualizan y mejoran su práctica asistencial a pacientes paliativos según la situación social que correspon$\mathrm{da}^{2}$.

Dentro de los contenidos competenciales de formación continuada en cuidados paliativos para enfermería, podemos distinguir diferentes aspectos como son las habilidades de comunicación en las etapas de duelo, el manejo de los síntomas y el dolor, el abordaje de la espiritualidad teniendo en cuenta las diferencias culturales de los pacientes y sus familias, así como el empleo de cuidados complementarios durante el avance de la enfermedad2, 3 . El alcance de estas habilidades va a depender del nivel de capacitación del profesional implicado, y de integrar un enfoque holístico que promueva reforzar los valores y deseos de la persona y su familia, así como su dignidad y autonomía en la toma de decisiones responsables de su proceso terminal ${ }^{4}$.

En el aprendizaje de los cuidados en fin de vida, se ha de tener en cuenta la reflexión personal de las emociones, y el desarrollo de intervenciones compasivas, donde la relación con el paciente se transforme en el elemento principal ${ }^{5}$. Esta actitud, viene influenciada por experiencias personales (edad, raza, religión, actitudes hacia la muerte), experiencias pasadas (nivel de formación, formación en tanatología) y profesionales (experiencia como enfermera y experiencia con pacientes en fase terminal) 6 .

Para definir los contenidos de una actividad formativa, desde una perspectiva multidisciplinar, debemos considerar los siguientes principios: globalidad, significancia (pertinencia), construcción, alternancia, aplicación, interacción, integración, distinción, coherencia y transferencia7. Por otro lado una competencia se define como un "saber-actuar complejo, que comprende la movilización y la combinación eficaz de recursos internos y externos al interior de una familia de situaciones"8. Para ello se requieren conocimientos y comportamientos que son aprehendidos a través de la actividad. Este concepto no está ligado solamente a la adquisición de saberes declarativos (qué), sino también a la integración de 
saberes prácticos (cómo) y condicionales (cuándo y porqué) construidos además de por la acción, por una secuencia de acciones de carácter social, afectivo y experimental. Así, el término competencia se caracteriza por ser integrador, combinatorio, con capacidad de desarroIlo, contextual y evolutivo?.

En cuanto a la adquisición de competencias, las diferentes corrientes psicopedagógicas como el empirismo, el interaccionismo, el conductismo, el constructivismo y el cognitivismo, influyen en la creación y trasformación del conocimiento ligado a las ciencias enfermera$\mathrm{s}^{10}$. La corriente constructivista y socio constructivista, en sus dos acepciones, biológica y social, es la que más se adapta al enfoque didáctico de "aprender a aprender", en la que el aprendizaje se considera un proceso progresivo, y el alumno, el eje principal de su desarrollo. Según, J. Piaget (constructivismo biológico) el individuo integra el conocimiento en función de las herramientas cognitivas internas de que dispone (inteligencia). Por otro lado, L.Vigotsky (constructivismo social) toma en consideración las interacciones sociales, que son las principales fuentes de interpretación de conocimiento.

En la actualidad, las herramientas pedagógicas empleadas en enfermería compatibles con la formación por compe- tencias, vienen influenciadas por el incremento en el acceso a la tecnología y a la formación en línea. De este modo, son consideradas como innovadoras, el empleo de la simulación o los juegos de rol en el laboratorio, o bien el uso del arte o la narrativa basada en problemas, cuyo fundamento en ambos casos es el desarrollo de la práctica reflexiva ${ }^{11}$.

El objetivo general de este trabajo es la construcción y el diseño de un curso de formación continuada en cuidados paliativos para enfermería. Los objetivos específicos, son mejorar la formación continuada de enfermería en cuidados paliativos, repercutiendo en su desarrollo profesional, según el contexto clínico y a través de una perspectiva multidisciplinar. También se pretende fomentar la participación constructiva entre el alumnado, el análisis, la reflexión y la discusión de los contenidos del curso, mediante el empleo de herramientas pedagógicas adecuadas para tal fin, y a través de un espíritu de colaboración y codesarrollo intragrupo.

\section{MetodologíA}

Para el diseño del curso se ha empleado el referencial de competencias de la Facultad de Enfermería de la Universidad de Montreal (UdM-Canadá), que sigue el modelo de atención de cui- 
dados de la teoría del Caring12. Esta estructura, se contempla para todos sus programas de formación y se basa en los términos cuidado, continuidad, comunidad, comunicación, rigor científico, interdisciplinariedad, liderazgo, humanismo y profesionalismo $^{13}$ (tabla1). Estos criterios, cumplen con los objetivos y exigencias pedagógicas de los alumnos, y favorecen las destrezas adquiridas según un determinado campo. El estudiante aprende el modelo de cuidados por medio de la acción, de un modo gradual y según sus necesidades.

En el diseño del curso, se han seguido una serie de pasos que se describen a continuación:

1. Determinar las competencias a alcanzar.

2. Determinar el grado de desarrollo y profundización de las competencias al término de la actividad.

3. Determinar los recursos internos a movilizar (conocimientos, actitudes, conductas).

4. Escalamiento gradual de las competencias en el conjunto de la formación.

5. Determinar la modalidad pedagógica.

6. Determinar la organización de trabajo de los formadores y estudiantes.
7. Determinar la modalidad de evaluación al inicio y al final de la actividad.

Para la enseñanza de este modelo (Caring) desde un punto de vista epistemológico, se ha considerado el paradigma del aprendizaje, en contraposición con el antiguo paradigma de la enseñanza, que tiene en cuenta la formación interactiva de competencias ligadas a un portafolio determinado, y adquiridas a través de la transferencia interdependiente de conocimientos y la promoción de la autonomía y la reflexión activas del alumno ${ }^{8}$.

En la determinación de los recursos internos a movilizar, se han tenido en cuenta distintos programas educativos de países pioneros en cuidados paliativos como son ${ }^{14}$ :

Programas de formación de la Palliative Nursing Program de Canadá que prioriza el desarrollo de ${ }^{15}$ :

Aspectos filosóficos, historia del desarrollo del movimiento hospice y de los cuidados paliativos, trabajo interdisciplinar en equipo, trabajo con la familia, aspectos culturales y espirituales con respecto al fin de vida y de la muerte.

Desarrollo de tratamientos médicos, intervenciones enfermeras, terapias complementarias usadas en el cuidado del paciente con enfermedad terminal, control de síntomas, control del dolor. 
Intervenciones psicoterapéuticas.

Programa de formación de Reino Unido, centrado en los siete parámetros "C":

- Comunicación, Coordinación, Control de síntomas, Continuidad del cuidado, formación Continuada, Cuidados de soporte y Cuidados en la muerte ${ }^{16}$.

- Modelo de EEUU (Certified hospice and Palliative Nurse): advance practice Nursing (palliative care nurse practitioner program) que se basa en las competencias del programa ENELC17.

En base a estas premisas, se ha desarrollado un organigrama que refleja los horarios y el escalamiento de los contenidos del curso, y que tiene en cuenta las estrategias pedagógicas seleccionadas.

El perfil del alumnado de esta actividad de formación, va dirigido a enfermeras graduadas que desarrollan su actividad en cualquier ámbito profesional. El curso se recomienda para grupos de 15 a 20 personas y el material didáctico necesario para su desarrollo es disponer de una sala amplia con pizarra y material informático (ordenador y proyector). Para evaluar la adquisición de competencias, se ha diseñado un cuestionario de 12 preguntas que ha de realizarse al inicio y al final de la actividad.

Durante el desarrollo de la investigación, se ha realizado una búsqueda bibliográfica en las principales fuentes de datos disponibles (PubMed, Cochrane, Cuiden, CINHAL) acerca de las herramientas pedagógicas empleadas en enfermería compatibles con la formación por competencias. También se ha seguido un curso de posgrado relacionado con nuevas estrategias de formación en enfermería en la UdM.

Dicho proyecto ha sido aprobado por el Comité de Ética de la Universidad de Montreal, y supervisado por el centro de formación e innovación de enfermería (CIFI) del mismo centro.

\section{Resultados}

Según los pasos que se han desarrollado en el diseño del curso se han obtenido los siguientes resultados.

Los contenidos del curso desde una perspectiva de cuidados paliativos, se han estructurado en base a 4 competencias principales:

Ejercer un razonamiento clínico enfermero para contribuir al confort y la calidad de vida de los pacientes y familiares en situación de fin de vida.

Actuar con humanismo en el acompañamiento a la persona/familia en 
sus experiencias en fin de vida teniendo en cuenta su contexto cultural y moral.

Actuar con profesionalismo, teniendo en cuenta el código deontológico de la profesión para promover la autonomía y la libertad del paciente en la toma de decisiones conscientes a su situación de fin de vida.

Colaborar con el equipo multiprofesional y ejercer liderazgo, para asegurar la continuidad de los cuidados de la persona al final de la vida.

En la profundización de dichas competencias se emplea la tabla 2, que describe para cada una de ellas su grado de desarrollo.

El curso se compone de un total de 20 horas distribuido en cinco días, en horario de mañana (de 9:00 a 13:00h) o de tarde (de 16:00 a 20:00h). Cada día consta de 4 horas de formación con una pausa de 20 minutos cada día. El escalamiento de las competencias y su distribución, en el conjunto de la formación, se dispone en el siguiente cronograma según la tabla 3 :

El curso se divide en una parte teórica y una práctica. La parte teórica, emplea clases magistrales distribuidas según los días del curso, de modo que el día 1 (teoría 1) se realiza una introducción a la filosofía de los cuidados paliativos y a la normativa legal en materia de muerte digna ${ }^{18}$. El día 2(teoría 2) plantea un abordaje de signos y síntomas desde la perspectiva del dolor total y aspectos de emergencias en cuidados paliativos ${ }^{19}$. El día 3 (teoría 3) aborda contenidos de ética y comunicación en cuidados paliativos20. Y finalmente el día 4 (teoría 4), trata la pérdida y el duelo en estas circunstancias ${ }^{21}$. Además el estudio de un caso, describe una situación real o ficticia, en vistas a desarrollar una valoración, un diagnóstico, una planificación y una evaluación de cuidados, similares a los de la práctica clínica enfermera. Este método, se trabaja en pequeños grupos y se realiza una exposición grupal posterior. En este curso, se ha desarrollado un caso clínico ficticio, a través de una historia narrada dividida en tres partes. La parte 1 abarca conceptos relacionados con el manejo del dolor total en el ámbito de la hospitalización. La parte 2 se centra en conceptos relacionados con la comunicación y manejo del proceso de la enfermedad paliativa en el domicilio. La parte 3 se dirige al manejo de duelo y del proceso de muerte en el domicilio (últimos días). Algunos fragmentos de la narrativa del caso son:

Irene Amenabar es una mujer de 48 años, que en 2011 se sometió a una mastectomía radical del pecho izquierdo...está casada con Mario, un empleado de banca que trabaja en horario de oficina. Sus hijos, Adrián, Carlos y Sara 
de 22, 16 y 8 años estudian en la universidad y en el colegio respectivamente. No tienen más familia en la ciudad... En abril de 2015, acude a urgencias por cefalea intensa, sensación de falta de aire y pérdida de peso progresiva de dos meses de evolución. Dice tener muchas punzadas en la espalda... (parte 1) La primera visita del equipo sanitario que acontece en el domicilio, es cuatro días después del alta hospitalaria..."Mira que blanca estoy, que ojeras tengo, jestos tratamientos me están matando!.". (parte 2). Una mañana después del desayuno, Irene sufre un desvanecimiento que le deja inconsciente...Su marido nervioso, controla que respira adecuadamente, pero teme que pueda pasarle algo, teme que sufra. Afortunadamente, Adrián el hijo mayor estaba en casa y no había acudido esa mañana a la universidad, porque tenía al día siguiente un examen...Llaman inmediatamente al servicio de urgencias extrahospitalario que acude al domicilio... Silvia y Enrique acudieron al funeral de Irene; sentían hacerlo tras la relación profesional que habían desarrollado a lo largo del tiempo...(parte 3).

Los alumnos cada día, desarrollan la actividad del caso en dos etapas. En la primera etapa, tras realizar una lectura crítica del caso, conforman un mapa conceptual de los aspectos más importantes del relato, y lo discuten en grupos reducidos de 3-4 personas. Posteriormente se ponen en común los diferentes mapas conceptuales de los diferentes grupos, para sacar conclusiones de forma general (tabla 4).

Dichas conclusiones irán encaminadas a clarificar el rol de la enfermera en cuidados paliativos, así como los cuidados necesarios para el paciente y su familia. Esta metodología mejora el juicio clínico, desde un enfoque reflexivo y con una sólida autonomía y liderazgo profesional que repercute en la prestación de cuidados de alta calidad. El estudiante se apoya de la teoría adquirida esa misma jornada, y también a través de sus propios conocimientos. De este modo es capaz de analizar, construir e integrar nuevos contenidos con respecto al caso, que podrá aplicar en un futuro en su entorno laboral. Está basado en la técnica del aprendizaje por problemas adaptada a la duración y a los contenidos de este curso22.

Con respecto al desarrollo de actividades prácticas, estás van encaminadas a relacionar la teoría y la práctica profesional. Se enumeran las siguientes:

Actividad 1: Brainstorming (día 1): Mediante esta técnica, cada alumno en clase lee el texto "Competencias enfermeras en cuidados paliativos"2 y pos- 
teriormente, realiza una puesta en común grupal de los conceptos más relevantes.

Taller 1 (día 1): "La muerte una realidad profesional". Este taller es un ejercicio de reflexión personal, de escucha y de respeto por los valores éticos, tanto personales como sociales. Se compone de una introducción a la realidad de la muerte en el contexto profesional sanitario. Se empleará una metodología desestructurada en la que a través de preguntas y respuestas libres, se llegará a la generalidad del tema tratado. Se expondrán las preguntas de forma global de manera que se genere un debate con las ideas representadas.

Actividad 2 (día 2): Valoración del dolor total (físico, psicológico, social y espiritual) a través de un caso clínico, según diferentes escalas de valoración. Se presentan las siguientes escalas que harán referencia a la primera parte del caso de Irene Amenabar: escala de valoración del dolor, escala Edmonton de síntomas, escala Zarit reducida de claudicación familiar, escala de Intervención sistémica familiar, y la escala Palliative performance status (PPS).

Taller 2 (día 2). "Una noche en el hospital al cuidado de un paciente terminal". El objetivo del taller es comprender el significado atribuido por los enfermeros/as durante el turno de noche en el contexto del cuidado paliativo, en el intento de atender a las necesidades de los clientes y sus cuidadores ${ }^{23}$. De este modo, las experiencias profesionales de unos, pueden ayudar en el desarrollo y formación clínica de otros. Se realiza el taller en grupos reducidos de 3-4 personas.

Actividad 3: "Conversaciones en la práctica al final de la vida" (día 3). Las conversaciones se centran en fragmentos que versan sobre necesidades tanto físicas, como emocionales, y que surgen entre el paciente y el profesional de la salud. Pretende fomentar las herramientas de comunicación. El alumno lee los textos individualmente y luego se exponen las reflexiones de forma grupal.

Taller 3: "Terapias complementarias y mindfulness" (día3). En este taller, se realiza un acercamiento a las terapias complementarias o modalidades de cura alternativa, que se emplean en cuidados paliativos desde un punto de vista holístico. Inicialmente se realiza de forma práctica y en grupo la toma de conciencia de la respiración y la técnica de mindfulness dirigidas ${ }^{24}$. Por último, se realiza una práctica sobre masaje de manos y aromaterapia; y se visualiza un video al respecto. Cada ejercicio tiene una duración de 15 minutos.

Actividad 4: "Codesarrollo profesional en la limitación del esfuerzo tera- 
péutico" (día 4). En esta actividad, se hace referencia al artículo 20 de la Ley foral 8/2011, de 24 de marzo18, de derechos y garantías de la dignidad de la persona en el proceso de la muerte, que describe los deberes de los profesionales sanitarios con respecto a la limitación del esfuerzo terapéutico. Se lee el texto y se exponen de forma grupal aquellas situaciones personales y profesionales, vividas con respecto al tema tratado.

Taller 4: "El testamento vital y el documento de voluntades anticipadas"(día 4). La manifestación de voluntades anticipadas o instrucciones previas, constituye un modo de decidir de forma anticipada sobre los tratamientos y cuidados sanitarios futuros que se desean recibir cuando se ha perdido la capacidad de expresar la voluntad. En grupos reducidos se reflexiona sobre sus objetivos y consecuencias. Para ello, se hace referencia al caso de Irene Amenabar.

El quinto día del curso se distribuye con actividades de simulación y rol play. Estos son métodos que pretenden desarrollar habilidades prácticas y de comunicación a través de la reproducción de un modelo simplificado pero ajustado a la realidad 25 . Requieren la participación de un actor, que simula una situación, para la cual el participante ha de demostrar sus destrezas en su mane- jo clínico. El resto de participantes, funcionan como observadores y trasmiten su opinión de la escena posteriormente. Se permite cometer errores sin preocuparse en su repercusión, de manera que los participantes se vuelven más conscientes en reconocer e integrar las mejores habilidades prácticas. El rol del facilitador, que en este caso es el profesor, es organizar la simulación, regular la ansiedad, proporcionar claras instrucciones sobre el procedimiento, asegurarse el entendimiento de los participantes y favorecer la discusión y la reflexión de forma constructiva y equilibrada. La discusión o comentario posterior a la simulación (debriefing) se hace en grupos reducidos y guiados por una planilla de objetivos $^{26}$. Se desarrollan tres escenarios que hacen referencia a situaciones del manejo del duelo y la pérdida en el profesional de la salud, cuidados paliativos a Mario, un paciente pediátrico, y sobre la necesidad espiritual de María, que simula una situación de sufrimiento espiritual en un paciente terminal.

Tanto al iniciar como al finalizar el curso se realizará una evaluación del curso por parte de los alumnos, así como una prueba objetiva de 12 preguntas tipo test, 4 por cada competencia relacionada con el curso. 


\section{DISCUSIÓN}

Debido a los cambios sociodemográficos y sociopolíticos de la actualidad, los profesionales de enfermería deberán atender a personas que sufren enfermedades crónicas (cáncer, cardiorrespiratorias y neurológicas), que después de recibir el tratamiento de su situación aguda/terminal en el medio hospitalario, se trasladan al domicilio donde precisarán de un cuidado regular ${ }^{27}$. La falta de acceso a unos cuidados paliativos de calidad, promueve el debate ético de proporcionar por derecho moral los siguientes aspectos $28:$ una cultura de cuidado, adecuada a los cambios sociodemográficos actuales; una mayor formación y traslación de competencias y conocimientos profesionales, basados en la evidencia; un mejor apoyo a los cuidadores, mediante programas de asistencia que contemplen el consejo en el duelo y el apoyo económico necesarios, una mejora de la accesibilidad y la coordinación territorial, y un sentido de liderazgo individual y comunitario en cuanto a la gestión de programas en cuidados paliativos.

Las motivaciones que nos llevan a realizar una formación continuada, tienen relación directa con nuestros intereses profesionales y personales ${ }^{29}$. El aprendizaje de las competencias en enfermería se centra en el alumno, el cual está influenciado por el entorno, la cultura, la inteligencia y por los métodos empleados en el proceso pedagógico, que han de ser debidamente evaluados ${ }^{30}$. Realizar una formación continuada en cuidados paliativos, va a favorecer en una mejora de la introspección y la autoeficacia de los profesionales en el manejo de las competencias en su práctica diaria ${ }^{31,32 .}$

La percepción de las enfermeras españolas en el manejo del paciente paliativo se centra más en el cuidado físico y no tanto en el cuidado emocional, social o espiritual33. Por otro lado, el estudio de Ballesteros et al refleja 4 aspectos fundamentales hacia lo que puede encaminarse la formación en cuidados paliativos como son, fomentar una visión integral de la disciplina enfermera, la importancia de la interacción con el paciente, la auto-reflexión personal en cuanto al concepto de muerte y su importancia en el currículum profesional34.

Los recursos internos a movilizar en este curso van encaminados a mejorar la formación continuada de enfermería en cuidados paliativos, siendo de especial interés para el desarrollo y mejora de la carrera profesional. Así mismo se pretende aumentar la significación de las competencias de enfermería en cuidados paliativos, según el contexto de trabajo, el tipo de patología y desde una perspec- 
tiva multidisciplinar y fomentar la participación constructiva en las sesiones de casos clínicos mediante el análisis, la reflexión y la discusión de los contenidos, y el empleo de las herramientas adecuadas para su formación. Además, se requiere compartir los conocimientos y experiencias profesionales propias en fin de vida con un espíritu de colaboración y codesarrollo profesional.

Debido a la poca participación de los alumnos en las sesiones plenarias en las que el profesor dicta el contenido teórico a los alumnos, en este curso se recomienda el empleo complementario de estrategias pedagógicas como son el estudio de caso, la simulación clínica y el taller de codesarrollo profesional. Este último es una variante de trabajo en grupo donde los participantes comparten sus experiencias aprendiendo los unos de los otros sobre un tema concreto. Todas estas técnicas, están demostrando tener beneficios individuales para los alumnos, y empleadas correctamente, tienen el potencial de promover el aprendizaje y la enseñanza atractiva y significativa en la formación continuada35. Además de promover el pensamiento crítico en enfermería, invitan a la creación de mapas conceptuales que refuerzan y organizan los conocimientos del profesional, así como a una mejor planificación de los cuidados en relación con su práctica clínica ${ }^{36}$. Por otro lado, el rol del profesor es esencial para trasmitir los conocimientos, además de ser clave como organizador, animador, y mediador del aprendizaje del alumno. En la adquisición de competencias hay que considerar la ética en que se fundamentan dichos conocimientos, así como los indicadores de evaluación que se identifican como idóneos para el contexto donde se desarrollen ${ }^{37}$.

Las instituciones sanitarias han de facilitar la realización de programas de formación continuada, como parte de la trayectoria profesional de los profesionales de enfermería.

\section{CONCLUSIONES}

Los enfoques educativos que se emplean en ciencias de la salud, no son estáticos, sino que están determinados por el contexto histórico en donde se transforman y renuevan a lo largo del tiempo. Éstos, han de integrarse en la cultura predominante, influenciados por los avances tecnológicos y sociopolíticos. Junto a la actualización del conocimiento es necesaria la construcción de unas competencias cognitivas que estén ligadas a dichas necesidades. En el diseño de las actividades y de los talleres en este curso, se ha tenido en cuenta por un lado que estén dirigidas al 
desarrollo profesional de la enfermería y por otro lado a los aspectos del binomio paciente-familia. La dinámica de este modelo, contribuye al desarrollo de competencias y de aptitudes que cumplen con los objetivos y exigencias de los alumnos.

El empleo de estas estrategias, proporciona la oportunidad de practicar nuevas herramientas y de experimentar con nuevas formas didácticas y de trabajo en grupo. Por otro lado, proporcionan un feedback constructivo, siendo una importante parte del proceso pedagógico, ya que se desarrollan áreas de más interés entre los participantes.

Este curso emplea diferentes líneas pedagógicas que promueven la relación profesor-estudiante a través de la creatividad, la autonomía, la autorresponsabilidad, la equidad, la cooperación y el respeto, y favorecen así, tanto el desarrollo personal y profesional. Se prevé un trabajo en equipo, así como la reflexión personal y el análisis práctico de las metodologías empleadas. De este modo, la figura del facilitador no se contempla como experto, sino como aquel que fomenta la actitud de curiosidad y respeto por cada experiencia de los participantes. Profesores y alumnos trabajan juntos para alcanzar los objetivos del taller de forma estructurada y flexible, promoviendo el intercambio de autoconocimiento siempre dirigido.

\section{AGRADECIMIENTOS}

Al Centro de Innovación y Formación de Enfermería (CIFI) de la Facultad de Enfermería de la Universidad de Montreal, a su directora Jacinte Pepin y a los alumnos del centro que han acompañado mi estancia formativa. 


\section{BIBLIOGRAFÍA}

1. De Vlieger M, Gorch N, Larkin P, Porchet F. A guide for the development of palliative Nurse Education in Europe. Report. Milán: Asociacion Europea de cuidados paliativos. Instituto Europeo de tumores, Palliative Nurse Education; 2004

2. Codorniu N, Guanter L, Molins A, Utor L. Competencias enfermeras en cuidados paliativos. 2013. http://www.secpal.com//Documentos/Blog/archivo_287.pdf.

3. Hernández Ruipérez T, Adánez Martínez MdG, Díaz Agea JL, García Pérez B, Leal Costa C. Diseño y validación de un modelo pedagógico basado en la simulación clínica dirigido a la formación de enfermería en el sistema de triaje estadounidense Emergency Severity Index. Emergencias. 2015 jun; 27(3): p. $155-160$

4. Codorniu N, Bleda M, Alburquerque E, Guanter L, Adell J, García F, et al. Cuidados enfermeros en Cuidados Paliativos: Análisis, consensos y retos. Index Enferm. 2011 ene.-jun; 20(1).

5. Brien LA, Legault A, Tremblay N. Affective learning in end-of-life care education: the experience of nurse education and students. Int J Pall Nurs. 2008; 14(12): p. 610-614.

6. Dunn KS, Otten C, Stephens E. Nursing Experience and the Care of Dying Patients. Oncol Nurs Forum. 2005 Jan; 32(1): p. 97-104

7. Lasnier F. Réussir la formation par competénces Montreal: Guérin; 2000.

8. Tardif J. Développer un programme par compétences: de l'intention à la mise en œuvre. Pédagogie collégiale. 2003 Mars; 16(3): p. 36-44.

9. Tardif J. Chapitre 1. In Tardif J. Le concept de compétence. Montreal: Chenelière Éducation; 2006. p. 17-51.

10. Donnadieu B, Genthon M, Vial M. Les théories de l'apprentissage. Quel usage pour les cadres de santé? Paris: Masson; 1998.

11. Crookesa K, Crookesb P, Walshb K. Meaningful and engaging teaching techniques for student nurses: A literature review. Nurse Educ Pract. 2013 Jul; 13(4): p. 239-43.

12. Marriner A. Modelos y teorías de enfermería Madrid: Rol; 1989.

13. Centre d'innovation en formation infirmière (CIFI). Référentiel de compétences des infirmières des communautés Premières Nations du Québec. [Online]; 2017 [cited 2019. Available from: https://www.cifi.umontreal.ca/documents/Publications/CIFI_Brochure_Referentiel_Competence_2017_web.pdf.

14. Utor Ponce L. Capacitación de enfermería en cuidados paliativos. Med Pal. 2007; 14(2): p. 100-103.
15. Canadian Hospice Palliative Care Association. [Online]; 2019. Available from: http://www.chpca.net/ professionals/nurses.aspx.

16. NHS, Health Education England. E-learning for healthcare. [Online]; 2019. Available from: https:// www.e-lfh.org.uk/programmes/end-of-life-care/.

17. HPNA, Hospice and palliative Nurses Association. Advancing Expert care. E-learning. [Online]; 2019. Available from: https://advancingexpertcare.org/elearning.

18. Gobierno de Navarra. Ley foral $8 / 2011$, de 24 de marzo, de derechos y garantías de la dignidad de la persona en el proceso de la muerte. [Online]; 2016 [cited 2016. Available from: http://www.lexnavarra.navarra.es/detalle.asp? $r=12315$

19. Tai S, Lee C, Wu C, Hsieh H, Huang J, Huang C, et al. Symptom severity of patients with advanced cancer in palliative care unit: longitudinal assessments of symptoms improvement. BMC Palliat Care. 2016 Mar 11; 15(1):32. 2016 Mar; 15(1): p. 32

20. Wittenberg-Lyles E, Goldsmith J, Platt C. Palliative care communication. Semin Oncol Nurs. 2014 Nov; 30(4): p. 280-86.

21. Tranter S, Josland E, Turner K. Nurse's bereavement needs and attitudes towards patient death: a qualitative descriptive study of nurses in a dialysis unit. J Ren Care. 2016; 42(2): p. 101-6.

22. Faculty of Nursing University of Montreal. L'apprentissage par situations infirmières cliniques (APSIC) au baccalauréat en sciences infirmiéres. 2004. Guide practique à l'intention des étudiantes et des étudiants.

23. Miranda da Silva M, Chagas Moreira M, Luzia Leite J, Lorenzini Erdmann A. El trabajo nocturno de la enfermería en el cuidado paliativo oncológico. Rev. Latino-Am. Enfermagem. 2013 mayo-jun; 21(3): p. 07 pantallas.

24. Scheick D. Developing self aware-mindfulness to manage countertransferance in the nursing-client relationship: an evaluation and developmental study. Journal of Professional Nursing. 2011 March-April; 27(2): p. 114-123.

25. Chamberland G, Lavoie L, Marquis D. Formules pédagogiques Quebec Pdld, editor. Paris: Eska; 1995.

26. Levett-Jones T, Lapkin S. A systematic review of the effectiveness of simulation debriefing in health professional education. Nurse Education Today. 2014; 34: p. e58-e63.

27. Coudray MA, C G. Le défi des competences. Comprendre et mettre en ouvre la réforme des études infirmières: Masson; 2009.

28. Carstairs S. Raising the bar: a roadmap for the future of palliative care in Canada. Ottawa: Senate of Canada; 2010. 
29. Mandicó García S. Reflexiones sobre la formación continuada de los profesionales de Enfermería en el Principat d'Andorra. Aglnf. 2015; 75(19): p. 128.

30. Bransford JD, Brown AL, Cocking RR. How People Learn. Brain, Mind, Experience, and School. Expanded ed. Washington: National academy press; 2004.

31. Adriaansen M, van Achterberg T, Borm G. Effects of a postqualification course in palliative care. Journal of advanced nursing. 2005; 49(1): p. 96 - 103.

32. Goulda D, Kellyb D, White S, Glen S. The impact of commissioning processes on the delivery of continuing professional education for cancer and palliative care. Nurse Education Today. 2004; 24: p. 443-451.

33. Arantzamendi M, Addington-Hall J, Saracibar M, Richardson A. Spanish nurses' preparedness to care for hospitalised terminally ill patients and their daily approach to caring. InternJ of Palliat Nurs. 2012; 18(12).

34. Ballesteros M, Centeno C, Arantzamendi M. A qualitative exploratory study of nursing students' assessment of the contribution of palliative care learning. Nurse Educ Today. 2014 Jun; 34(6): p. e1-6.

35. Kuebler KK. The Need for Continuing Nursing Education in Palliative Care and the Management of Symptomatic Chronic Disease. Georgia Nursing. 2010 Nov; 70(4): p. 9.

36. Toofany S. Critical thinking among nurses. Nursing management. 2008 Feb; 14(9): p. 28-31.

37. Schroeter K. Competency and credentialling institute. [Online]; 2008 [cited 2016 Mar 29. Available from: http://www.cc-institute.org/home. 


\section{ANEXOS Y TABLAS}

Tabla 1: Referencial de competencias de la Universidad de Montreal para el grado de enfermería.

1. Actuar con humanismo según una perspectiva disciplinar.

2. Ejercitar un razonamiento clínico enfermero.

3. Asegurar la continuidad de cuidados.

4. Favorecer una población en salud.

5. Tratar toda actividad profesional y disciplinar con rigor científico.

6. Actuar con profesionalismo.

7. Colaborar con los equipos profesionales.

8. Ejercer un liderazgo clínico en la práctica profesional enfermera.

Tabla 2: Profundización de las competencias determinadas para el curso.

\section{Competencia 1: Ejercer un razonamiento clínico}

1.1 Participar en la evaluación continua de las necesidades del paciente y su familia según los criterios establecidos y las herramientas válidas.

1.2 Elaborar un plan de cuidados individualizado, basado en los estándares holísticos del dolor total, y revisar regularmente el nivel de intervención, según los criterios de calidad de vida deseados por el paciente y su familia.

1.3 Ofrecer los tratamientos e intervenciones, encaminados al alivio del dolor y otros síntomas relacionados según la normativa establecida.

1.4 Conocer los patrones de enfermedad al final de la vida.

1.5 Anticipar los signos de fin de vida, así como las situaciones de emergencia y aplicar las medidas correspondientes.

1.6 Realizar los cuidados necesarios en el momento de la muerte.

1.7 Sostener la participación de los familiares en los cuidados del enfermo, respetando su voluntad de implicación y ofreciendo el apoyo requerido.

\section{Competencia 2: Actuar con humanismo}

2.1 Crear una relación compasiva y empática en la prestación de cuidados.

2.2 Establecer una relación de ayuda y asociación a la persona y su familia en todos los momentos del final de la vida a través de una comunicación fluida y continuada.

2.3 Respetar la dignidad de la persona en apoyo a toda acción sobre la calidad de vida, la esperanza y la voluntad definida por el paciente-familia. 
2.4 Abordar las necesidades espirituales (los valores, las creencias y otros aspectos culturales) relacionadas con el individuo y su familia.

2.5 Sostener al enfermo y su familia en la búsqueda de sentido en su experiencia en fin de vida.

2.6 Facilitar los procesos de adaptación a las situaciones presentes en fin de vida.

2.7 Dar soporte a los familiares durante la agonía y después del fallecimiento del paciente.

2.8 Promover intervenciones psicoterapéuticas en relación al duelo y la pérdida.

2.9 Respetar la puesta en práctica de rituales personalizados, relacionados con la pertenencia cultural del individuo y su familia.

\section{Competencia 3: Actuar con profesionalismo}

3.1 Respetar el curso de los acontecimientos, dilemas y reflexiones éticas en el contexto de fin de vida.

3.2 Implicar al enfermo y a sus familiares en los procesos de toma de decisiones.

3.3 Comenzar y mantener un diálogo con el paciente y sus familiares a cerca de sus voluntades al final de la vida, respecto a su cultura y criterios en relación a la calidad de vida.

3.4 Asegurarse que el enfermo y su familia disponen de información esencial que promueva la autonomía y la toma de decisiones en sus experiencias al final de la vida.

3.5 Revisar regularmente las voluntades del enfermo y de sus familiares en relación a los cuidados prestados.

3.6 Defender y representar los derechos y deberes de los pacientes y de los profesionales al final de la vida.

3.7 Asegurarse según la necesidad sentida, de la colaboración de las personas, recursos relacionados con una experiencia pertinente en fin de vida. Recursos de apoyo al individuo/familia en la comunidad

\section{Competencia 4: Colaborar con el equipo multiprofesional y ejercer liderazgo.}

4.1 Reconocer los valores personales, sus capacidades y sus límites en lo que concierne a la vida, la muerte y el sufrimiento.

4.2 Integrar la filosofía de los cuidados paliativos a los valores personales. Aspectos filosóficos, historia del desarrollo del movimiento hospice y de los cuidados paliativos, trabajo interdisciplinar en equipo, trabajo con la familia

4.3 Saber coordinar los distintos servicios que puedan intervenir en el proceso de fin de vida. Coordinación

4.4 Comprender la necesidad del trabajo interdisciplinar en colaboración con otros profesionales de la salud, en el abordaje del paciente terminal.

4.5 Compartir adecuadamente la información dentro de una perspectiva de colaboración interprofesional y de continuidad de cuidados.

4.6 Preservar la integridad y cuidar de sí mismo, prevención de la fatiga compasional.

ENE, revista de enfermeria

1988-348X 
DISEÑO DE UN CURSO DE FORMACIÓN CONTINUADA EN CUIDADOS PALIATIVOS BASADO EN COMPETENCIAS

4.7 Ofrecer y aceptar el apoyo de los compañeros de trabajo.

4.8 Participar en el trabajo reflexivo del equipo de cuidados para ofrecer cuidados óptimos en salud.

4.9 Aplicar las nociones de seguridad y calidad, así como el empleo eficaz de los recursos disponibles en el contexto de los cuidados paliativos.

Tabla 3. Cronograma del curso y distribución de las competencias a alcanzar a lo largo del curso.

\begin{tabular}{|c|c|c|c|c|c|}
\hline $9: 00-13: 00$ & Lunes & Martes & Miércoles & Jueves & Viernes \\
\hline 9:00-9:45 & $\begin{array}{l}\text { Presentación del } \\
\text { curso y test de } \\
\text { evaluación }\end{array}$ & Teoría 2 & Teoría 3 & Teoría 4 & \multirow{5}{*}{$\begin{array}{c}\text { Simulación Clínica y Rol } \\
\text { play }\end{array}$} \\
\hline 9:45:10:45 & & \multicolumn{3}{|c|}{ Estudio de caso } & \\
\hline & & Parte 1 & Parte 2 & Parte 3 & \\
\hline $10: 45-11: 05$ & Pausa & Pausa & Pausa & Pausa & \\
\hline \multirow{2}{*}{$\begin{array}{c}11: 05-12: 00 \\
------- \\
\text { 12:00-13:00h }\end{array}$} & Actividad 1 & Actividad 2 & Actividad 3 & Actividad 4 & \\
\hline & Taller 1 & Taller 2 & Taller 3 & Taller 4 & $\begin{array}{l}\text { Fin curso y } \\
\text { Test de evaluación }\end{array}$ \\
\hline $\begin{array}{l}\text { Competencias } \\
\text { a desarrollar }\end{array}$ & $\begin{array}{l}\text { C1.4, C2.3, C3.1, } \\
\text { C3.6, C4.1, C4.2 y } \\
\text { C4.6 }\end{array}$ & $\begin{array}{l}\text { C1.1, C1.2, C1.3, } \\
\text { C2.6, C3.4, C3.7, } \\
\text { C4.3, C4.5, C4.7, } \\
\text { C4.8 y C4.9. }\end{array}$ & $\begin{array}{l}\text { C1.5, C1.7, C2.1, } \\
\text { C2.2, C2.4, C3.2, } \\
\text { C3.3, C4.3, C4.4, } \\
\text { C4.5 y C4.6. }\end{array}$ & $\begin{array}{l}\text { C1.6, C1.7.C2.5, } \\
\text { C2.7, C2.8, C2.9 } \\
\text { у C3.5 }\end{array}$ & $\begin{array}{l}\text { C1.1, C1.5, C1.6, C1.7, } \\
\text { C2.1, C2.1, C2.5, C2.7, } \\
\text { C3.1, C3.5, C4.1, C4.4, } \\
\text { C4.7 y C4.9. }\end{array}$ \\
\hline
\end{tabular}

\section{Tabla 4: Resumen de la metodología del caso propuesta.}

1) Recogida de datos: se lee el caso y se recogen los datos que aparecen en el texto, resaltando los más importantes.

2) Análisis: Se plantean preguntas en relación a las necesidades que aparecen en el caso para enmarcar y aclarar la situación. (¿Qué, Porqué, Cómo, Cuándo, Desde...?)

3) Interpretación y esquematización: se realiza un esquema sobre la situación clínica y se realiza una hipótesis y una explicación de las intervenciones a realizar.

4) Puesta en común de los hallazgos y crítica de las propuestas realizadas. Será necesaria la asignación de un representante que exponga los resultados obtenidos por el grupo al que representa. 